\title{
Enabling multipath optical routing with hybrid differential delay compensation
}

\author{
Rodolfo Alvizu', ${ }^{1}$ Veronica Soto ${ }^{2}$ Sebastian Troia ${ }^{1}$, Guido Maier ${ }^{1}$ \\ ${ }^{1}$ Dipartimento di Elettronica, Informazione e Bioingegneria, Politecnico di Milano, 20133 Milan, Italy \\ ${ }^{2}$ Facultad de Ingeniería en Electrónica y Computación, Escuela Superior Politécnica del Litoral, Guayaquil, \\ Ecuador \\ e-mail:vsoto@espol.edu.ec, \{rodolfoenrique.alvizu, sebastian.troia, guido.maier\}@polimi.it
}

\begin{abstract}
Historically, Internet traffic has been routed over the shortest path: that was convenient for best-effort data traffic, but it is not always suitable for today's scenario where applications can require bandwidth higher than what is available in a single link, even when provided by an optical wavelength-channel. Multi-path (MP) routing is a network functionality that provides more capacity, reduces the probability of link congestion and increases the availability of the transport service.

This paper elaborates on techniques to mitigate the differential delay in all optical networks, recognized as the main problem of multi-path (MP) routing. This work shows how hybrid differential delay compensation (H-DDC) can greatly reduce the use of expensive reconstruction buffers in all optical networks implementing MP optical routing. A novel mixed integer linear programming formulation is proposed for the novel wavelength + H-DDC assignment problem: distributed fiber delay lines (FDL)s combined with electronic reconstruction buffers collocated at optical regeneration points. Numerical results based on commercially available (and rack mountable) FDLs demonstrate the effectiveness of H-DDC in medium size transport networks.
\end{abstract}

Keywords: Multipath routing, differential delay compensation, fiber delay line, all-optical networks, network optimization.

\section{INTRODUCTION}

Multimedia applications based on ultra-high definition digital media formats, virtual reality, immersive video, inter-datacenter communications, e-science applications can require bandwidth higher than what is available in a single link, even when the link is provided by an optical wavelength-channel. Moreover, with the advent of 5G, vertical services and the digital transformation, the transport optical network must be able to provide high standards of performance in terms of traffic delay and resilience, to support the coexistence of different $5 \mathrm{G}$ services in the same physical medium.

Internet traffic has been routed over a single shortest path, but this is not always suitable for today's scenario. Multipath (MP) routing is a network functionality that allows to split (or, if needed, to replicate) a data flow from a source to a destination over a set of multiple paths, and to reconstruct it as a single flow (or pick the best copy) at the destination. This technique provides more capacity, reduces the probability of link congestion and increases the availability of the transport service (when used for $1+1$ protection) [1]. The main obstacle that has limited the adoption of MP routing is that the sub-paths in a MP connection experience each one a specific delay, leading to a differential delay (DD) between them. DD introduces jitter and generates negative impact on customer services. One way to overcome this hitch is performing differential delay compensation (DDC), i.e. buffering the packets along the shortest of the paths to reconstruct the original data sequence. Normally, DDC is performed using reconstruction buffers at destination nodes, known as electronic-DDC (E-DDC). However, reconstruction buffers are expensive memories that need to work at twice the line rate and they could be a bottleneck when buffers overflow [1]. This gets worst in optical high-speed networks, where the high line rates impose faster and larger reconstruction buffers. A proposed method is to distribute the DDC (D-DDC) in transit nodes [2]. In all optical networks (AON)s, D-DDC is expensive due to the addition of Electro/Optical (E/O) and Optical/Electrical (E/O) conversions to perform D-DDC. D-DDC can be co-located with optical regenerators to avoid extra E/O/E [2], however this solution is limited by the geographical characteristics of the network. A novel approach for AONs is to use fiber delay lines (FDL)s to perform distributed transparent DDC (T-DDC), that does not require extra E/O/E conversions [3]. FDL modules available off-the-shelf introduce delay with coarse granularity. Therefore, we do not propose to totally eliminate electronic delay compensation, but to use FDLs and electronic buffers in a combination, in the so-called Hybrid-DDC (H-DDC). DDC can be collocated at optical regeneration points when FDLs are not enough to totally compensate all DD.

This work improves the optimization model presented [3] by considering the novel wavelength + H-DDC assignment problem with E-DDC collocated with the optical regeneration points. Moreover, the new model allows to evaluate a realistic scenario using T-DDC capabilities based on commercially available (and rack mountable) FDLs. Numerical results demonstrated the effectiveness of H-DDC in medium size transport networks. 


\section{WDM-BASED NETWORK WITH ELECTRONIC AND TRANSPARENT DDC CAPABILITIES}

This work focuses on WDM networks with hybrid DDC capabilities. Figure 1 illustrates the proposed node architecture. The switching matrix of the Optical Cross Connector (OXC) can be dynamically configured by a centralized control plane as in the case of Transport Software Defined Networking (T-SDN) [4]. The end-points of a wavelength channel are dropped (O/E conversion) and added (E/O conversion), like demands $d_{1}$ and $d_{2}$ in figure 1 . The OXC allows to perform optical bypass, creating lightpaths spanning across multiple nodes $\left(d_{4}\right.$ in figure 1). Due to degradation of optical signals along the lightpath, optical regeneration must be introduced for lightpaths longer than the regeneration span $\vartheta$. Optical regeneration is an electronic process comprising Reamplifying, Reshaping and Retiming (3R regeneration), performed by a pair of back-to-back transponders (O/E/O conversions). The proposed node architecture can perform distributed DDC electronically when regeneration is needed (like $d_{3}$ ), or transparently using up to M FDL modules (like $d_{5}$ and $d_{6}$ ).

\section{MILP FORMULATION}

We propose an optimization problem to plan a MP routing-capable optical network by applying H-DDC techniques. This problem consists in performing: MP-disjoint routing, wavelength assignment, optical regenerator placement, FDL-module placement for T-DDC, and dimensioning of E-DDC capacity at regeneration points. Due to its complexity, we divided the problem in two phases. MILP ${ }_{1}$ to solve the well-known DD-constrained MP routing problem (not presented in this work). MILP $_{2}$ to solve the novel wavelength $+\mathrm{H}$-DDC assignment problem, with E-DDC collocated with the optical regeneration points. Each demand $d \in D$ is described by: source and destination nodes, differential delay upper bound $\Delta_{d}$ and the required bandwidth $h_{d} . h_{d}$ is given in terms of number of channels, where we assume that each channel operates at $L$ Gbps. Tables 1 and 2 describe the input parameters and the variables of the proposed MILP formulation.

The objective function of $\mathrm{MILP}_{2}$ (1) minimizes the total propagation delay while reducing use of E-DDC, either centralized (at the end node) or distributed (collocated with regeneration points). $\alpha$ is a weighting factor, with $\alpha>1$. Solenoidality and fiber-capacity constraints are given by (2) and (3). Constraints (4) and (13) assign TDDC, while (5) computes total DDC of each path. Constraint (6) ensures that only one demand can use a specific wavelength of a FDL module. Constraints (7)-(9) establish that T-DDC can be performed only in the wavelength and nodes of the path used to serve a demand. Wavelength continuity constraint is given by (10). Constraints (11) and (12) limit the number of FDL module that a demand can use in a node. Constraints (14) and (15) establish the regeneration points according to link lengths and T-DDC from the source to each hop.

Table 1. MILP2 Input Parameters

\begin{tabular}{|c|l|}
\hline Symbol & Input Parameters Description \\
\hline$h_{d}$ & no. of L Gbps channels requested by demand d \\
\hline$W$ & number of wavelength channels per fiber \\
\hline$F_{e}$ & number of fibers in the link $e$ \\
\hline$\delta_{e d p}$ & 1 if link $e$ belongs to path $p$ of demand $d$ \\
\hline$\phi_{d}$ & max. delay of the MP set of demand $d$ (sec) \\
\hline$\alpha$ & cost of using electronic buffer for E-DDC $(\alpha>1)$ \\
\hline$G$ & Fiber length of FDL modules $\{60,90\}$ km \\
\hline$M$ & number of available FDL modules per node \\
\hline$x_{d p}$ & 1 if MILP1 chose path p to serve demand d \\
\hline$D_{d p}$ & total DDC in path p of demand d (sec) \\
\hline$\omega_{d p s}$ & length of path p until hop s for demand d (km) \\
\hline$\gamma_{d p s v}$ & $\begin{array}{l}1 \text { if node } \mathrm{v} \text { belongs to path p until hop s for } \\
\text { demand d }\end{array}$ \\
\hline$\pi_{d p s e}$ & $\begin{array}{l}1 \text { if link e belongs to path p until hop s for demand } \\
\text { d }\end{array}$ \\
\hline$k_{v d p}$ & 1 if node v is in the path p of demand d \\
\hline$\beta_{e v}$ & 1 if link e is an outgoing link of node v \\
\hline$\vartheta$ & optical regeneration span (km) \\
\hline
\end{tabular}

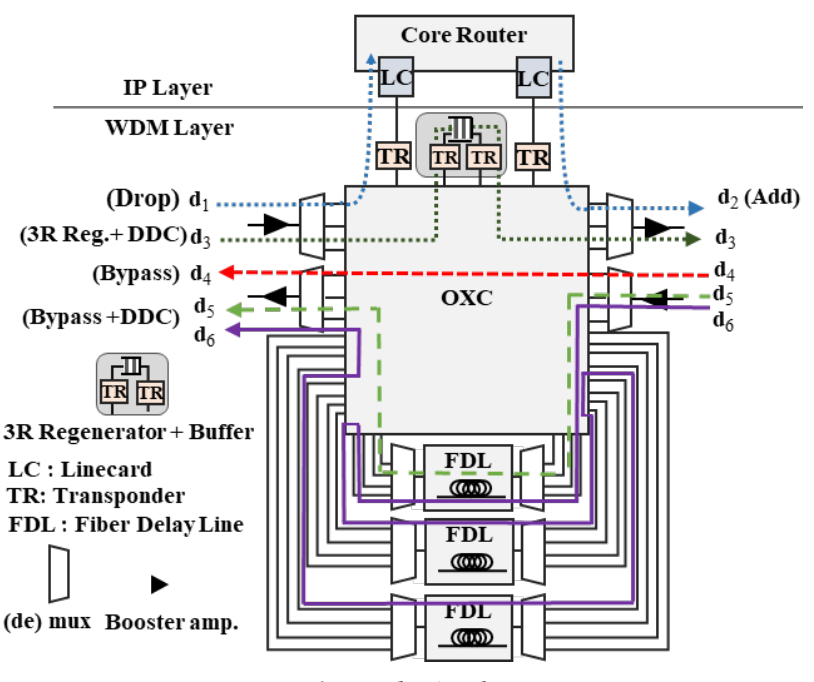

Figure 1. Node Architecture

Table 2. MILP2 Variables

\begin{tabular}{|c|l|}
\hline Symbol & Variables Description \\
\hline$X_{d p \lambda}^{\prime}$ & 1 if wavelength $\lambda \in W$ is used by path $p \in P$ of demand $d$ \\
\hline$Z_{d p v m \lambda}$ & 1 if wavelength $\lambda$ of FDL-module $m \in M$ at node $\mathrm{v}$ is used to compensate path p of demand d \\
\hline$T_{d p}$ & amount of T-DDC compensated in path p of demand d (sec) \\
\hline$B_{d p}^{d i s t}$ & amount of Distributed E-DDC compensated in path $p$ of demand $d$ (sec) \\
\hline$B_{d p}^{\text {cent }}$ & amount of Centralized E-DDC compensated in path $p$ of demand $d$ (sec) \\
\hline
\end{tabular}




$$
\begin{aligned}
& \min \sum_{d \in D} \sum_{p \in P_{d}}\left(T_{d p}+\alpha B_{d p}^{\text {dist }}+\alpha B_{d p}^{\text {cent }}\right) \\
& \text { Subject to: } \quad \sum_{p \in P_{d}} \sum_{\lambda \in W} X_{d p \lambda}^{\prime}=h_{d}, d \in D \\
& \sum_{d \in D} \sum_{p \in P_{d}} \delta_{e d p} X_{d p \lambda}^{\prime} \leq F_{e}, e \in E, \lambda \in W \\
& \sum_{v \in V} \sum_{m \in M} \sum_{\lambda \in W} Z_{d p v m \lambda} G \leq T_{d p}, d \in D, p \in P_{d} \\
& T_{d p}+B_{d p}^{d i s t}+B_{d p}^{c e n t}=D_{d p}, \mathrm{~d} \in D, \mathrm{p} \in P_{d} \\
& \sum_{d \in D} \sum_{p \in P_{d}} Z_{d p v m \lambda} \leq 1, v \in V, m \in M, \lambda \in W \\
& Z_{d p v m \lambda} \leq X_{d p \lambda}^{\prime}, d \in D, p \in P_{d}, v \in V, m \in M, \lambda \in W \\
& X_{d p \lambda}^{\prime} \leq x_{d p}, d \in D, p \in P_{d}, d \in D, \lambda \in W \\
& Z_{\text {dpvm }} \leq k_{v d p}, d \in D, p \in P_{d}, v \in V, m \in M, \lambda \in W \\
& \sum_{\lambda \in W} X_{d p \lambda}^{\prime} \leq 1, d \in D, p \in P_{d} \\
& \sum_{m \in M} \sum_{\lambda \in W} Z_{d p v m \lambda} \geq \beta_{e v} k_{v d p} \rho_{e d p}, \mathrm{e} \in E, d \in D, p \in P_{d}, v \in V \\
& \rho_{e d p} \leq M, e \in E, d \in D, p \in P_{d} \\
& \sum_{e \in E} \rho_{e d p} G=T_{d p}, d \in D, p \in P_{d} \\
& x_{d p} \omega_{d p s}+\sum_{e \in E}\left(\Pi_{d p s e} \rho_{e d p} G\right) \leq \sum_{v \in V}\left(\vartheta R_{d p v} \gamma_{d p s v}\right)+\vartheta, d \in D, p \in P_{d}, s \in S_{d p} \\
& R_{d p v} \leq x_{d p} k_{v d p}, d \in D, p \in P_{d}, v \in V \\
& X_{d p \lambda}^{\prime}, Z_{d p v m \lambda}, R_{d p v} \in \text { binary; } B_{d p}^{\text {dist }}, B_{d p}^{\text {cent }}, T_{d p} \in R^{+} ; \rho_{e d p} \in Z^{+}
\end{aligned}
$$

\section{PERFORMANCE EVALUATION}

The proposed model has been tested using the network topologies described in Figure 2: i) NSFNET with average link length greater than regeneration span A $>\vartheta$ (typically, $\vartheta=1200 \mathrm{~km}$ ), considered as a large-size network [5]. ii) Polish and German topologies, with $\mathrm{A}<\vartheta$, considered as medium-size networks [5,6]. The larger the link length, the larger the DDC to be performed, making it harder to use MP-disjoint routing.

Figure 3 presents the E-DDC reduction gain achieved with the proposed H-DDC, with respect to the amount of T-DDC capacity installed in each node of the network. We assumed two possible alternative lengths for the FDL modules: 60 and $90 \mathrm{~km}$, which can be fitted into a rack mount unit [7]. For the medium-size networks, the gain reached a saturation point at more than $90 \%$ of E-DDC reduction for $\mathrm{M}>3$ and $\mathrm{G}=60$ and $\mathrm{G}=90 \mathrm{~km}$. For NSFNET (large-size), the gain curves increased linearly with the T-DDC capacity installed in the nodes. The use of larger FDL modules with G>100 km can be a good solution for large networks (such as NSFNET), however in this work we restrain to rack-mountable components.

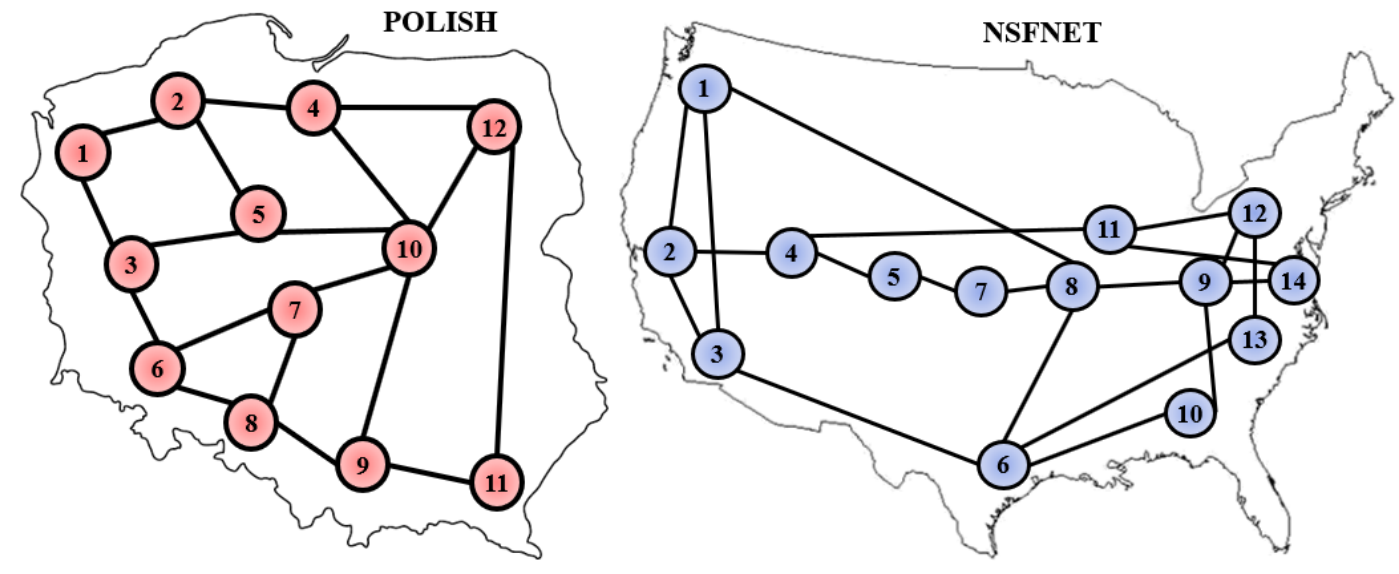

\begin{tabular}{cccccc}
\hline Networks & Nodes (V) & Links $(\mathrm{E})$ & Min. Link Length $(\mathrm{km})$ & Max. Link Length $(\mathrm{km})$ & Avg. Link Length $(\mathrm{km})$ \\
\hline POLISH & 12 & 18 & 69 & 353 & 189,2 \\
GERMAN & 17 & 26 & 36 & 353 & 170,3 \\
NSFNET & 14 & 21 & 312 & 3408 & 1299,1 \\
\hline
\end{tabular}

Figure 2. Network topologies used for performance evaluation 


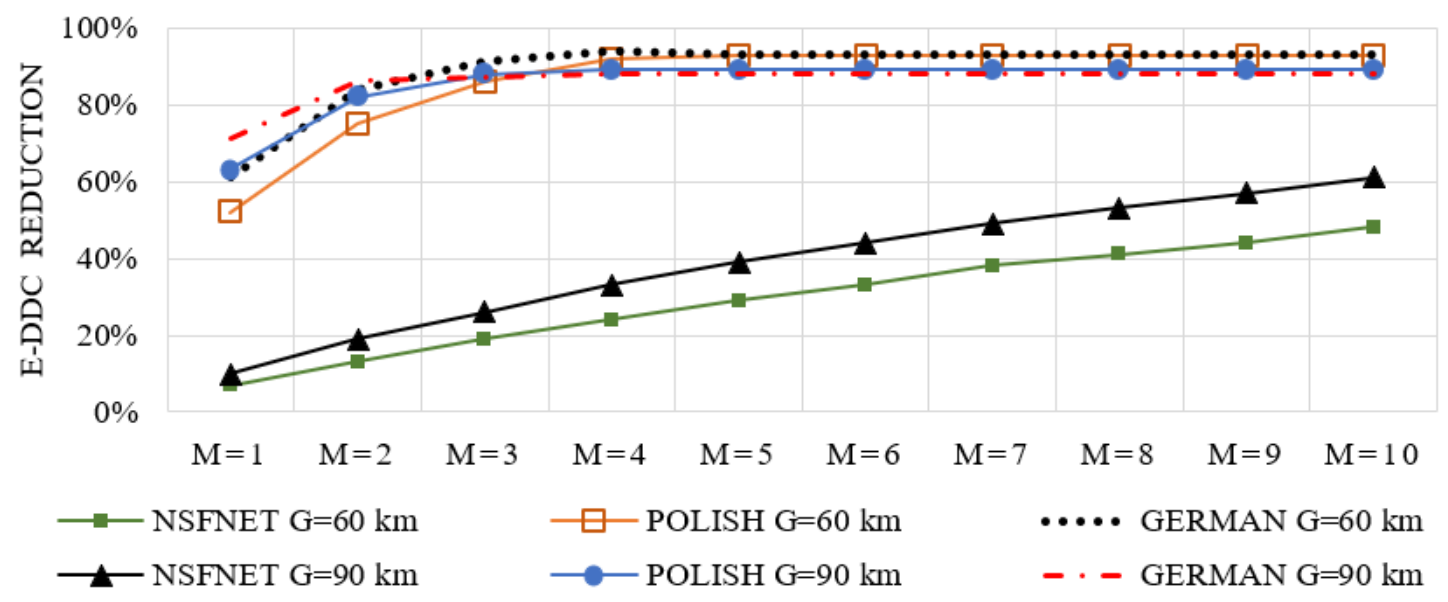

Figure 3. Overall E-DDC reduction when varying the max. number of FDL modules per node (M)
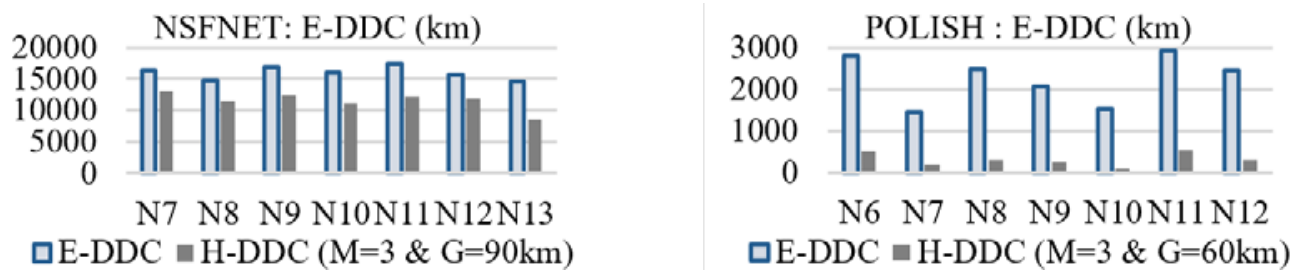

Figure 4. Electronic vs Hybrid DDC

Figure 4 displays the E-DDC performed at a sub-set of the nodes, using kilometers of E-DDC as a measure. For a medium-size network (such as Polish), the great reduction of E-DDC when using H-DDC is clearly visible in the right inset of Figure 4. The left inset of Figure 4 presents the results for the large-size NSFNET using $\mathrm{G}=90 \mathrm{~km}$ and $\mathrm{M}=3$.In this case, the amount of effectively-reduced E-DDC is larger than in the medium-size network, but the reduction is smaller due to larger average link length.

\section{CONCLUSIONS}

This paper analyzes the use of H-DDC approach for optical MP routing that effectively reduces the electronic buffers and unnecessary O/E/O conversions by using transparent compensation with FDLs. The proposed MILPbased matheuristic shows the benefits of using FDLs to reduce the differential delay, which is the main problem of MP routing. Based on the numerical results, the method achieved a reduction gain of more than $90 \%$ for European national networks such as the Poland and Germany cases. Moreover, the model is the first attempt to jointly optimize the assignment of T-DDC, wavelength and regeneration points where E-DDC can be used without introducing extra $\mathrm{O} / \mathrm{E} / \mathrm{O}$ conversions.

\section{ACKNOWLEDGEMENTS}

The work leading to these results has been supported by the European Community under grant agreement no. 761727 Metro-Haul project

\section{REFERENCES}

[1] A. Anurag, et al, "Differential delay aware routing for Ethernet over SONET/SDH,” in IEEE INFOCOM, vol. 2, Mar. 2005, pp. 1117-1127.

[2] J. Santos, et al., "Optical transport network design with collocated regeneration and differential delay compensation,” in IEEE HPSR, June 2012, pp. 204-209.

[3] R. Alvizu, et al, "Multipath optical routing with compact fiber delay line-based differential delay compensation," NOC, 2016, pp. 58-63.

[4] R. Alvizu et al., "Comprehensive survey on T-SDN: Software-defined Networking for Transport Networks," in IEEE Communications Surveys \& Tutorials, vol. PP, no. 99, pp. 1-1.

[5] A. Betker et al., "Reference transport network scenarios," MultiTeraNet Report, July 2013.

[6] S. Orlowski et al., "Sndlib 1.0 survivable network design library,” Networks, vol. 55, no. 3, pp. 276-286, 2010.

[7] Rack mount fiber optic delay lines. [Online]. Available: http://www.fiberplus.com/dloption8.html 